\title{
Food Texture Quantification of Tempura Using Magnetic Food Texture Sensor and Time-series Data
}

\author{
Ninomae Souda, ${ }^{1}$ Hiroyuki Nakamoto, ${ }^{1 *}$ Futoshi Kobayashi, ${ }^{1}$ \\ Yuya Nagahata, ${ }^{2}$ and Yoriyasu Hirosue ${ }^{2}$ \\ ${ }^{1}$ Graduate School of System Informatics, Kobe University, \\ 1-1 Rokkodai-cho, Nada-ku, Kobe, Hyogo 657-8501, Japan \\ ${ }^{2}$ J-Oil Mills, Inc., 8-1,Akashi-cho, Chuo-ku, Tokyo 104-0044, Japan
}

(Received February 22, 2019; accepted May 22, 2019)

Keywords: sensor, food texture, time-series data, dynamic time warping, tempura

Food texture is one of the characteristics of food and is an important factor in food development. In this paper, a method of food texture quantification based on expressions for texture instead of physical properties is proposed. The method obtains two sets of time-series data of force and vibration during food compression using a magnetic food texture sensor. Dynamic time warping (DTW) matches the time-series data and standard data and calculates the degree of texture similarity. The standard data are determined by DTW barycenter averaging (DBA). The experimental results for tempura showed that the degree of texture similarity based on the vibration data had a strong correlation with the sensory evaluation data.

\section{Introduction}

Recently, improvements in food processing technology have enabled the production of foods with delicate and complex food textures. Food texture is an important factor for consumers as well as taste and flavor. In addition, aging societies such as Japan require food textures that contribute to health through chewing and safe swallowing. Food processing industries and food supply chains therefore need to evaluate and design food textures. ${ }^{(1)}$

The texture profile analysis (TPA) proposed by Szczesniak has been widely used as a typical evaluation method for food texture. ${ }^{(2)}$ In TPA, the force applied during food fracture is measured as a time series using a measurement instrument such as a texture analyzer and physical properties such as hardness, adhesiveness, and cohesiveness are determined from the time series of the force. However, these physical properties cannot explain delicate and complex food textures. The measurement of force only is not enough to evaluate food texture. It is necessary to improve the measurement instrument and the evaluation method for food texture.

Some researchers have reported evaluation methods for food texture that use acoustic sensors. Acoustic studies can be roughly separated into two approaches. One deals with air-conducted food sound and the other deals with bone-conducted food sound. Both sounds contribute to our perception of food texture. ${ }^{(3)}$ Evaluation methods that combine acoustic and

*Corresponding author: e-mail: nakamoto@panda.kobe-u.ac.jp

https://doi.org/10.18494/SAM.2019.2342 
force measurements during food fracture have been developed. Chen et al. recorded force, displacement, and acoustic signals simultaneously by attaching an acoustic envelope detector to a texture analyzer. They used the results to evaluate the crispness of six kinds of biscuits. ${ }^{(4)}$ Taniwaki and Kohyama simultaneously measured acoustic and mechanical events during the deformation and fracture of potato chips using a texture analyzer and a microphone, and evaluated the crispness from each characteristic. ${ }^{(5)}$ Vickers reported that the combination of acoustic and forcedeformation measurements more appropriately describes foodtexture characteristics than either technique only. ${ }^{(6)}$ Srisawas and Jindal analyzed the crispness of snack food products based on the frequency domain spectrum of the acoustic patterns during sample fracture. In addition, they classified snack foods using neural network models. ${ }^{(7)}$ On the other hand, several evaluation methods that use elements other than sound have been reported. Kohyama et al. used a multiple-point sheet sensor to detect the pressure distribution during food fracture and directly determined the textural differences among crackers. ${ }^{(8)}$ Shibata et al. developed a robotic sensing system that quantitatively evaluates the mechanical and geometrical characteristics of texture by measuring the pressure distribution of gel-like foods. ${ }^{(9)}$ These studies mainly analyzed data for which the correlation between the measurement data and the sensory characteristics was strong, however, a few studies dealt with direct food-texture quantification from measurement data.

In this paper, we propose a food texture quantification method based on expressions for food texture. A food texture sensor simultaneously measures time series of force and vibration during food fracture. Using this quantification method, we calculate the degree of texture similarity between time-series data and reference data by dynamic time warping (DTW) to obtain a food texture value. Experimental results confirm the potential of the proposed method.

\section{Materials and Methods}

\subsection{Food texture sensor}

The sensor consists of a plunger, an elastomer, a base, and a sensor board. Figure 1 shows the structure of the sensor. The plunger has a wedge shape and includes a permanent magnet inside it. The elastomer is a soft layer made from a urethane material. The plunger and elastomer are placed on a base. The sensor board has sensing elements on the bottom surface, which are eight magnetoresistive elements and two inductors. The measurement principle of the sensor is as follows. When the sensor fractures a food sample with the top of the wedge, the plunger is moved down to compress the food. Simultaneously, the magnetic field applied by the permanent magnet to each sensing element changes. The magnetoresistive elements detect the magnetic field strength and the inductors detect the change in magnetic flux density as an induced voltage. A desktop PC records the data obtained at a sampling frequency of $10 \mathrm{kHz}$. In addition, the $\mathrm{PC}$ calculates the force from the data obtained from the magnetoresistive elements using a multiple regression equation and deals with the induced voltage as the vibration during the fracture. ${ }^{(10)}$ The sensor is attached to a motorized stage as shown in Fig. 2. 

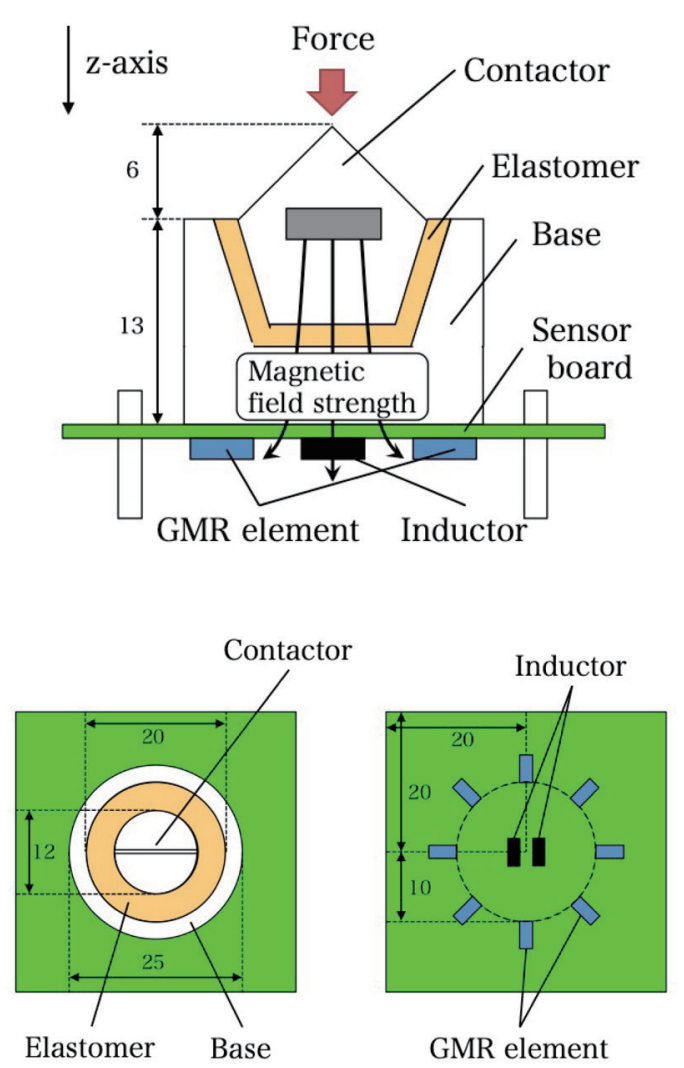

Fig. 1. (Color online) Structure of magnetic food texture sensor.

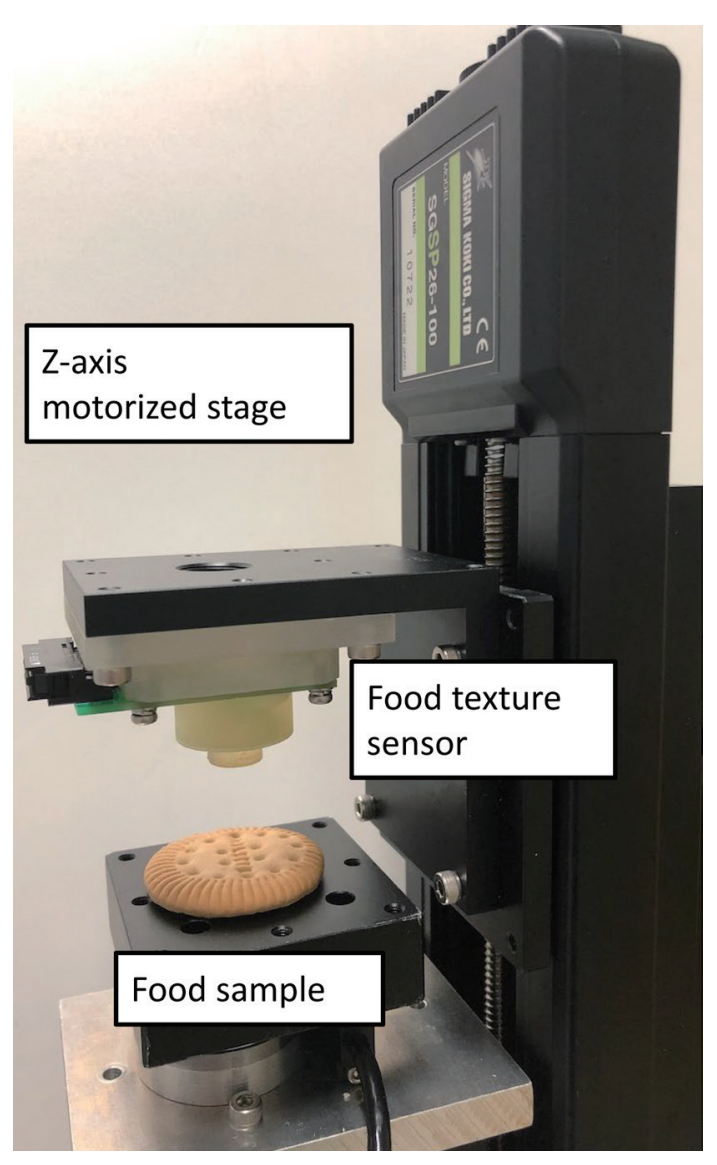

Fig. 2. (Color online) Measurement system with food texture sensor.

\subsection{Quantification method}

The sensor obtains time-series data of force and vibration. From the time-series data of force, TPA is carried out to determine profiles of hardness, cohesiveness, and so on. On the other hand, in this study, we propose a quantification method using DTW. DTW is an algorithm used to calculate the DTW distance between two sets of time-series data and is widely used in the areas of speech and image recognition..$^{(11-13)}$ The DTW distance expresses the degree of dissimilarity. In the calculation, DTW simultaneously determines the optimal match between the two sets of time-series data. Even if the time-series data of force and vibration vary along the time axis, DTW calculates the minimal DTW distance from the optimal match. In addition, DTW deals with all the time-series data not some of them.

DTW calculates the DTW distance between measurement data and standard texture data. The standard texture data must include a characteristic waveform of the target food texture. In this study, DTW barycenter averaging (DBA), which is an averaging algorithm using DTW, determines the standard data from some data. ${ }^{(14)}$ DBA determines the optimal match between 
two sets of time-series data selected from some measurement data by DTW. On the basis of the matching results, DBA calculates barycenters from the matched measurement data and determines the barycenter data as averaged data. This study uses the averaged data as the standard texture data. DBA determines the standard texture data of force from some measured time-series data of force. However, the time-series data of vibration have a waveform with negative and positive spikes around $0 \mathrm{~V}$ and are not suitable for DTW. Hence, we converted the time-series data of vibration to time-series cumulative sum data. ${ }^{(15)}$ The cumulative sum data have a waveform rising to the right, which is suitable for DTW.

In addition, the DTW distance is the degree of dissimilarity and has different ranges for force and vibration data. In this study, to avoid confusion and normalize both force and vibration, the degree of texture similarity $s$ is defined using the following equation:

$$
s=\frac{d_{\max }-d}{d_{\max }-d_{\min }} \times 100
$$

where $d$ is the DTW distance, and $d_{\max }$ and $d_{\min }$ are the maximal and minimal DTW distances, respectively. $d_{\max }$ and $d_{\min }$ for force and vibration are determined individually. The degree of texture similarity ranges between 0 and 100 .

\subsection{Experiments}

A professional cook fried tempura samples individually for $2 \mathrm{~min} 30 \mathrm{~s}$ and placed them on a tray for $5 \mathrm{~min}$ at room temperature. After that, the system compressed each sample twice by reciprocating motion that imitates the action of the jaw, and measured the time-series data of force and vibration. The height of the sample was about $20 \mathrm{~mm}$. The system began the compression motion at the position it first came in contact with the sample and compressed it to a height of $15 \mathrm{~mm}$ at a velocity of $3.0 \mathrm{~mm} / \mathrm{s}$. The number of samples was eight. Hence, the system recorded eight time-series data of force and vibration. We performed cross-validation using four of the data to obtain the standard texture data and the other four data to calculate the degree of texture similarity.

In addition, we performed a sensory evaluation of the tempura samples using the visual analog scale, which is a method of evaluating various sensations as points on straight lines. ${ }^{(16)}$ We provided a panel of seven experts with 100-mm-long lines with "weak" written at the left end and "strong" written at the right end. In the sensory evaluation, we used three expressions for texture in Japanese, Sakusaku, Karikari, and Paripari, which have similar meanings to crispy and crunchy in English. The panels determined the definition of each texture based on a discussion as follows.

- Sakusaku: crunchiness with moderate hardness (rhythmically chewable hardness).

- Karikari: crunchiness with chewy hardness.

- Paripari: crispness of firm and thin foods. 


\section{Results}

Table 1 shows the sensory evaluation values, which are the average values given by the seven panel members. We determined a threshold value for the sensory evaluation of 60 and extracted the food texture expressions from Table 1. The underlined values in Table 1 were extracted by comparison with the threshold. For example, samples $\mathrm{B}$ and $\mathrm{C}$ have the food texture of Karikari. Then, DBA was used to determine the standard data for each expression for food texture. The standard data for Karikari were determined from the time-series data of samples $\mathrm{B}$ and $\mathrm{C}$.

Figure 3 shows typical time-series data of force and vibration. The dashed and black lines show the force and vibration data, respectively. The horizontal axis shows time. The left and right vertical axes show the force and vibration voltage, respectively. Figure 4 shows the standard texture data of the force and the cumulative sum of the vibration.

The degree of texture similarity of each sample is shown in Table 2. For comparison with the degree of texture similarity, Table 2 also shows the sensory evaluation value. In addition, we calculated the correlation coefficients between the degree of texture similarity and the sensory evaluation value. Figures 5(a) and 5(b) show the correlations for each expression for texture and for each sample, respectively.

\section{Discussion}

From Fig. 3, sample $\mathrm{C}$ had the strongest force and sample $\mathrm{D}$ had the weakest. In addition, sample $\mathrm{C}$ had the highest maximum value and occurrence frequency of vibration, whereas sample D had no vibration at all. Sample A had a strong force and frequent vibration values, and sample B had frequent vibration. According to the sensory evaluation shown in Table 2, sample D, which had a weak force and vibration, also had low values for the three expressions for texture. Hence, we consider that the perception of the food texture requires a certain force and vibration. The sensory values of Karikari and Paripari were highest for sample $\mathrm{C}$ and second highest for sample B. We tend to perceive these two textures from a strong force and frequent vibration. On the other hand, the sensory value of Sakusaku for sample C was small, and sample B, having the highest sensory value of Sakusaku, did not have a particularly strong force. In the case of a strong force, the value of Sakusaku decreases. The time-series data of force and vibration express features of the food texture of tempura made from different starches.

Table 1

Sensory evaluation values.

\begin{tabular}{lccc}
\hline Sample & Sakusaku & Karikari & Paripari \\
\hline A & $\underline{61}$ & 41 & 40 \\
B & $\underline{64}$ & $\underline{64}$ & 53 \\
C & 35 & $\underline{81}$ & $\underline{69}$ \\
D & 25 & 15 & 14 \\
E & 48 & 53 & 32 \\
\hline
\end{tabular}




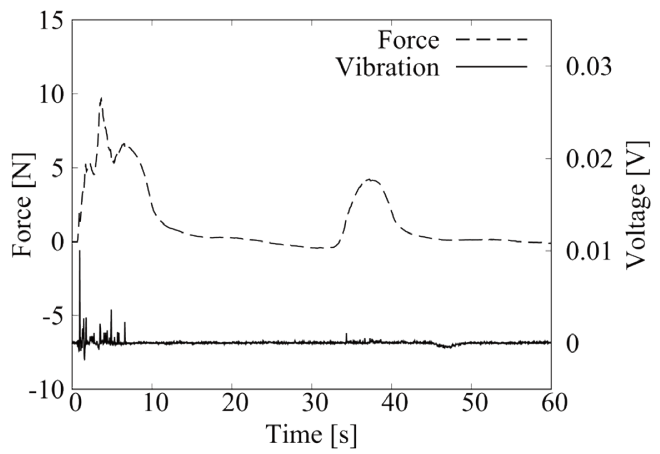

(a)

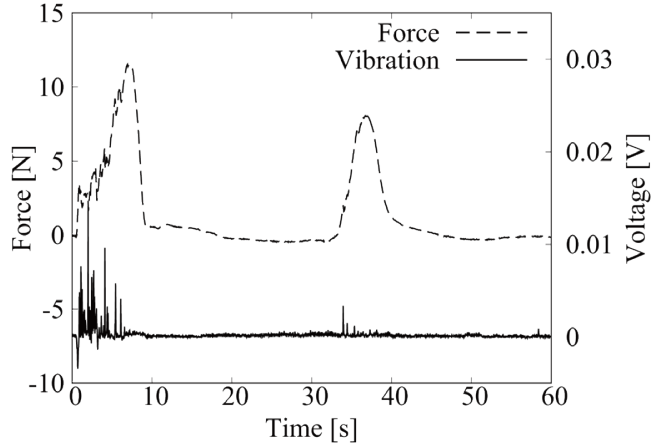

(c)

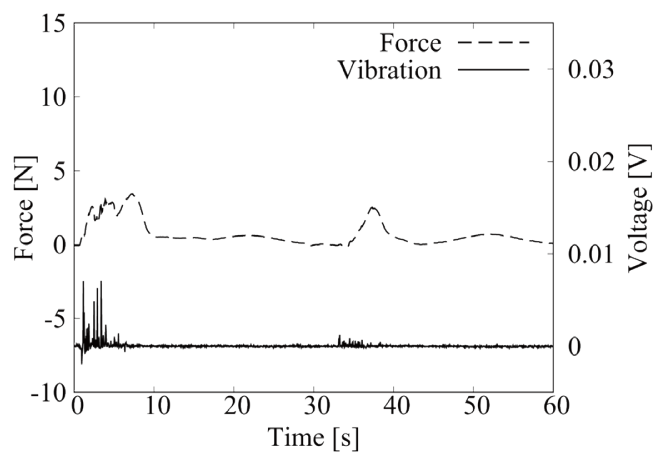

(b)

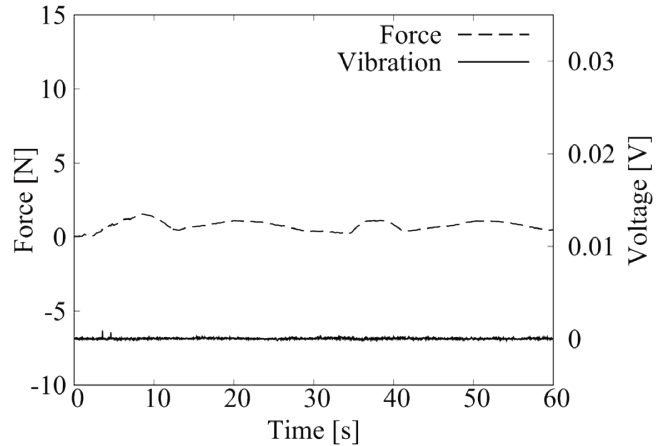

(d)

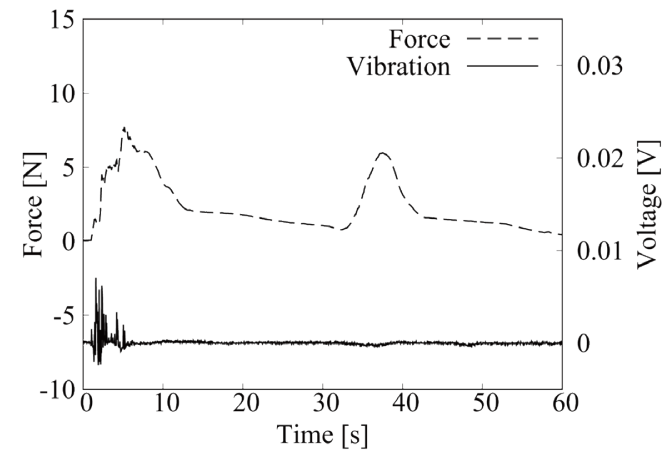

(e)

Fig. 3. Examples of time series of force and vibration. (a) Sample A, (b) Sample B, (c) Sample C, (d) Sample D, and (e) Sample E.

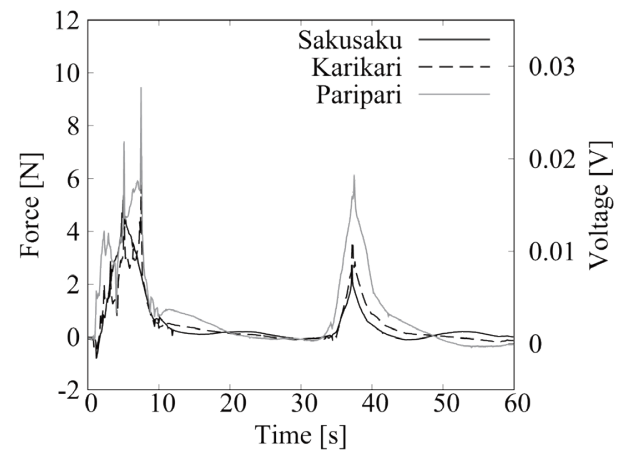

(a)

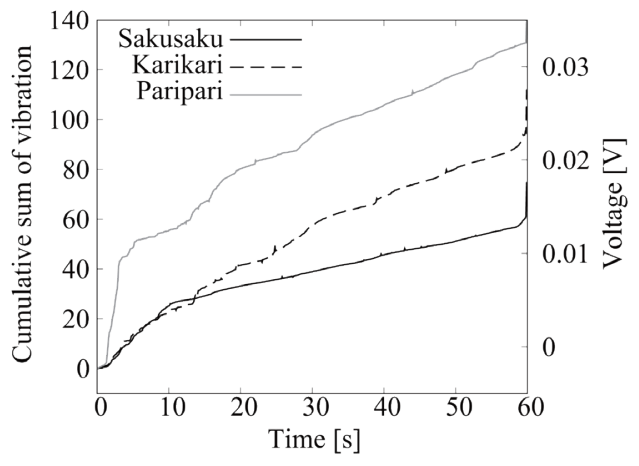

(b)

Fig. 4. Standard time-series data. (a) Force and (b) Cumulative sum of vibration. 
Table 2

Degree of texture similarity and sensory evaluation value (F: force, V: vibration, S: sensory).

\begin{tabular}{|c|c|c|c|c|c|c|c|c|c|}
\hline \multirow{2}{*}{ Sample } & \multicolumn{3}{|c|}{ Sakusaku } & \multicolumn{3}{|c|}{ Karikari } & \multicolumn{3}{|c|}{ Paripari } \\
\hline & $\mathrm{F}$ & V & S & $\mathrm{F}$ & V & $\mathrm{S}$ & $\mathrm{F}$ & V & $\mathrm{S}$ \\
\hline A & 84 & 97 & 61 & 85 & 86 & 41 & 81 & 53 & 40 \\
\hline B & 79 & 98 & 64 & 82 & 88 & 64 & 67 & 58 & 53 \\
\hline $\mathrm{C}$ & 79 & 86 & 35 & 88 & 96 & 81 & 91 & 94 & 69 \\
\hline D & 78 & 83 & 25 & 79 & 67 & 15 & 53 & 26 & 14 \\
\hline $\mathrm{E}$ & 87 & 95 & 48 & 88 & 87 & 53 & 85 & 57 & 32 \\
\hline
\end{tabular}

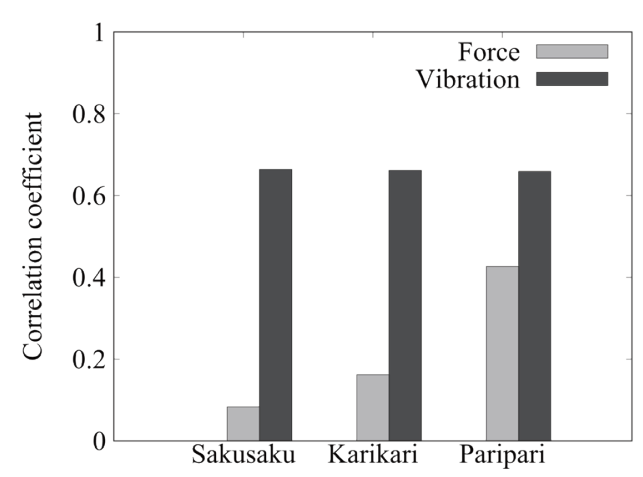

(a)

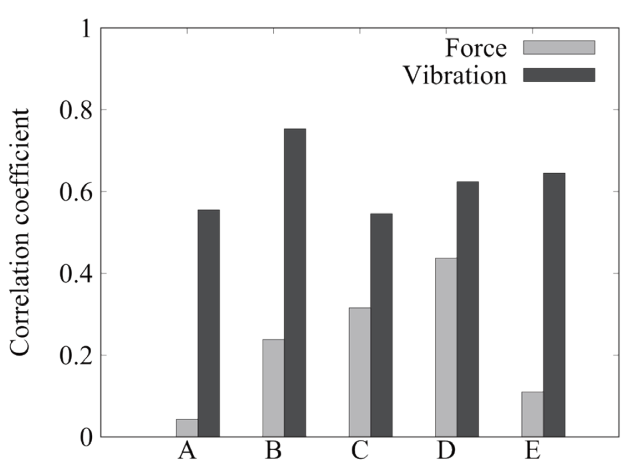

(b)

Fig. 5. Correlation coefficients between degree of texture similarity and sensory evaluation value. (a) Texture and (b) sample.

In Fig. 4, the standard texture data were different for Sakusaku, Karikari, and Paripari. The standard data of Karikari showed a strong force at the first compression, and the Paripari data showed a strong force at the second compression. The standard data show that food with the Karikari texture tends to lose this texture after the first compression in comparison with Paripari food. Regarding the cumulative sum of vibration, the standard data of Paripari were high in comparison with those of Sakusaku and Karikari. In addition, the standard data of Sakusaku were lower than those of Karikari. Hence, the standard data obtained by DBA express the difference between the three food textures.

Table 2 shows that sample E had the highest degree of texture similarity to Sakusaku in terms of force and sample B had the highest degree in terms of vibration. Regarding Sakusaku, the vibration had the same tendency in terms of the degree of texture similarity and the sensory evaluation value. On the other hand, the force showed a significantly different tendency. Regarding Karikari, there was little difference in force among the five samples. Sample B had the highest value for vibration and Sample $\mathrm{C}$ had the lowest value. These results agree with the tendency of the sensory evaluation values. The results for Paripari had a considerably different tendency from those for the other textures. Sample $\mathrm{C}$ had the highest force and vibration and sample D had the lowest values, similarly to that observed in the sensory evaluation. 
If the correlation coefficient ranges from 0.7 to 1.0, the relationship has a strong correlation. In Fig. 5(a), the force of Paripari and the vibration of all textures had a medium-strength positive correlation. In Fig. 5(b), the force of sample D and the vibration of all samples had a medium-strength and a more positive correlation and the force of samples $\mathrm{B}$ and $\mathrm{C}$ had a weak positive correlation. Since the correlation between the degree of texture similarity and the sensory evaluation was positive for the three expressions for crispness, this result means that the proposed method using DTW and DBA has a potential to quantify food texture on the basis of the expressions for texture. In particular, the correlations of vibration with the three textures were stronger than those of force. Hence, the vibration data include the major characteristics of the three textures of tempura. We consider that the cumulative sum of vibration is effective for texture quantification.

Regarding the limitations of this study, eight sets of force and vibration data were obtained and we obtained the standard texture data from them. We need to obtain more data to generalize the standard data. In addition, this study focused on the food texture of crispness. The vibration data had strong correlations with the three expressions for crispness. On the other hand, the force data did not have strong correlations. We need to verify the proposed method for other food textures using the characteristics of both force and vibration.

\section{Conclusions}

A magnetic food texture sensor with two sensing elements obtained the time series of force and vibration data for five tempura samples. The standard data of three textures were obtained from the time-series data by DBA. DTW determined the degree of texture similarity between standard data and measurement data. The degree of texture similarity for vibration had a particularly strong correlation with the sensory evaluation data.

In this study, the number of data used to obtain standard data was limited. It is necessary to obtain more data to generalize the standard data. In addition, we will verify the effectiveness of the proposed method for different textures and foods.

\section{Acknowledgments}

This work was supported by JSPS KAKENHI Grant Number JP16K00813.

\section{References}

1 A. R. Linnemann, E. M. T. Hendrix, R. Apaiah, and T. A. J. S. V. Boekel: NJAS - Wageningen J. Life Sci. 72-73 (2015). https://doi.org/10.1016/j.njas.2014.10.002

2 A. S. Szczesniak: Food Qual. Preference 13 (2002) 4. https://doi.org/10.1016/S0950-3293(01)00039-8

3 C. Dacremont: J. Texture Stud. 26 (1995) 1. https://doi.org/10.1111/j.1745-4603.1995.tb00782.x

4 J. Chen, C. Karlsson, and M. Povey: J. Texture Stud. 36 (2005) 2. https://doi.org/10.1111/j.1745-4603.2005.00008. $\mathrm{x}$

5 M. Taniwaki and K. Kohyama: J. Food Eng. 112 (2012) 4. https://doi.org/10.1016/j.jfoodeng.2012.05.015

6 Z. M. Vickers: J. Food Sci. 52 (1987) 1. https://doi.org/10.1111/j.1365-2621.1987.tb13990.x

7 W. Srisawas and V. K. Jindal: J. Texture Stud. 34 (2003) 4. https://doi.org/10.1111/j.1745-4603.2003.tb01072.x 
8 K. Kohyama, M. Nishi, and T. Suzuki: J. Food Sci. 62 (1997) 5. https://doi.org/10.1111/j.1365-2621.1997. tb15007.x

9 A. Shibata, A. Ikegami, M. Nakauma, and M. Higashimori: Robotics 6 (2017) 37. https://doi.org/10.3390/ robotics6040037

10 D. Nishikubo, H . Nakamoto, F. Kobayashi, and F. Kojima: Nippon Shokuhin Kagaku Kogaku Kaishi 63 (2016) (in Japanese). https://doi.org/10.3136/nskkk.63.268

11 H. Sakoe and S. Chiba: IEEE Trans. Acoustics, Speech, Signal Process. 26 (1978) 1. https://doi.org/10.1109/ TASSP.1978.1163055

12 T. B. Amin and I. Mahmood: 2008 2nd Int. Conf. Advances in Space Technol. (2008). https://doi.org/10.1109/ ICAST.2008.4747690

13 T. M. Rath and R. Manmathe: Proc. 2003 IEEE Comput. Soc. Conf. Comput. Vision and Pattern Recognit. (2003). https://doi.org/10.1109/CVPR.2003.1211511

14 F. Petitjean, A. Ketterlin, and P. Gancarski: Pattern Recognit. 44 (2011) 3. https://doi.org/10.1016/ j.patcog.2010.09.013

15 P. H. Ellaway: Electroencephalogr. Clin. Neurophysiol. 45 (1978) 2. https://doi.org/10.1016/00134694(78)90017-2

16 H. M. McCormack, D. J. Horne, and S. Sheather: Psychol. Med. 18 (1988) 4. https://doi.org/10.1017/ S0033291700009934 (1007\$-\$1019) 